\title{
Superconductor-proximity effect in chaotic and integrable billiards
}

\author{
J. A. Melsen, P. W. Brouwer, K. M. Frahm, and C. W. J. Beenakker \\ Instituut-Lorentz, University of Leiden \\ P.O. Box 9506, 2300 RA Leiden, The Netherlands
}

(To be published in Physica Scripta)

\begin{abstract}
We explore the effects of the proximity to a superconductor on the level density of a billiard for the two extreme cases that the classical motion in the billiard is chaotic or integrable. In zero magnetic field and for a uniform phase in the superconductor, a chaotic billiard has an excitation gap equal to the Thouless energy. In contrast, an integrable (rectangular or circular) billiard has a reduced density of states near the Fermi level, but no gap. We present numerical calculations for both cases in support of our analytical results. For the chaotic case, we calculate how the gap closes as a function of magnetic field or phase difference.
\end{abstract}

PACS numbers: 74.50.+r, 05.45.+b, 73.23.Ps, 74.80.Fp

There exists a way in quantum mechanics to distinguish classically chaotic systems from integrable systems, by looking at correlations between energy levels [1,2]. In a billiard with integrable dynamics, on the one hand, the energy levels are uncorrelated, and the spectrum has Poisson statistics. In a chaotic billiard, on the other hand, level repulsion leads to strong correlations and to Wigner-Dyson statistics [3]. Although the level correlations are different, the mean level density does not distinguish between chaotic and integrable billiards.

In a recent paper 顿 we have shown that the proximity to a superconductor makes it possible to distinguish chaotic from integrable billiards by looking at the density of states. In a chaotic billiard, on the one hand, we have found (using random-matrix theory) that the coupling to a superconductor by means of a point contact opens a gap in the density of states of the order of the Thouless energy $E_{\mathrm{T}}=N \Gamma \delta / 2 \pi$. Here $N$ is the number of transverse modes in the point contact, $\Gamma$ is the tunnel probability per mode, and $\delta$ is half the mean level spacing of the isolated billiard. In an integrable rectangular billiard, on the other hand, the density of states vanishes linearly near the Fermi level, without a gap. We have argued (using the Bohr-Sommerfeld approximation) that the absence of an excitation gap is generic for integrable systems. In these Proceedings we present numerical calculations for a chaotic billiard in support of the randommatrix theory, and we consider the effects of a magnetic field perpendicular to the billiard. We also present calculations for an integrable circular billiard, both exact and in the Bohr-Sommerfeld approximation.

The system studied is shown schematically in the inset of Fig. 1. A billiard consisting of a normal metal (N) in a perpendicular magnetic field $B$ is connected to two superconductors $\left(\mathrm{S}_{1}, \mathrm{~S}_{2}\right)$ by narrow leads, each containing $N / 2$ transverse modes at the Fermi energy $E_{\mathrm{F}}$. The order parameter in $\mathrm{S}_{1}$ and $\mathrm{S}_{2}$ has a phase difference $\phi \in[0, \pi]$. Mode $n$ couples to a superconductor with phase $\phi_{n}=\phi / 2$ for $1 \leq n \leq N / 2, \phi_{n}=-\phi / 2$ for $1+N / 2 \leq n \leq N$. For simplicity, we assume in this paper that there is no tunnel barrier in the leads $(\Gamma=1)$. (The generalization to $\Gamma \neq 1$ is straightforward $\left[\begin{array}{|c}\text {.) } \\ \Gamma\end{array}\right.$

The excitation spectrum of the billiard is discrete for energies $0<E<\Delta$, where $\Delta$ denotes the excitation gap in the bulk of the superconductors. We compute the spectrum starting from the scattering formulation of Ref. [5]. The billiard with the leads but without the superconductors has an $N \times N$ scattering matrix $S_{0}(E)$, at an energy $E$ relative to the Fermi level. The modes in the leads which form the basis of $S_{0}$ are chosen such that their wave functions are real at the NS interface. Evanescent modes in the leads are disregarded. The energy dependence of $S_{0}$ is set by the Thouless energy $E_{\mathrm{T}}$, which is inversely proportional to the dwell time of an electron in the billiard. Andreev reflection at the superconductors scatters electrons (at energy $E>0$ ) into holes (at energy $-E$ ), with a phase increment $-\phi_{n}-\arccos (E / \Delta)$. (Normal reflection at the superconductors can be neglected if $\Delta \ll E_{\mathrm{F}}$.) We assume that $E$ and $E_{\mathrm{T}}$ are both $\ll \Delta$, so that we may replace $\arccos (E / \Delta)$ by $\pi / 2$, while retaining the $E$-dependence of $S_{0}$. The excitation spectrum is obtained from the determinantal equation [5]

$$
\operatorname{Det}\left[1+S_{0}(E) e^{i \phi} S_{0}^{*}(-E) e^{-i \phi}\right]=0,
$$

where $\phi$ is a diagonal matrix with the phases $\phi_{n}$ on the diagonal.

The scattering matrix $S_{0}$ can be expressed in terms of the Hamiltonian matrix $H$ of the isolated billiard [6],

$$
S_{0}(E)=1-2 \pi i W^{\dagger}\left(E-H+i \pi W W^{\dagger}\right)^{-1} W .
$$

The dimension of $H$ is $M \times M$, and the limit $M \rightarrow \infty$ will be taken later on. The $M \times N$ coupling matrix $W$ has elements

$$
W_{m n}=\delta_{m n}\left(\frac{2 M \delta}{\pi^{2}}\right)^{1 / 2}, m=1,2, \ldots M, n=1,2, \ldots N
$$


The energy $\delta$ is one half of the mean level spacing of $H$. For the chaotic billiard, we assume that the Hermitian matrix $H$ has the Pandey-Mehta distribution [3,7]

$P(H) \propto$
$\exp \left(-\frac{M\left(1+\alpha^{2}\right)}{4 \lambda^{2}} \sum_{i, j=1}^{M}\left[\left(\operatorname{Re} H_{i j}\right)^{2}+\alpha^{-2}\left(\operatorname{Im} H_{i j}\right)^{2}\right]\right)$,

with $\lambda=2 M \delta / \pi$. The parameter $\alpha \in[0,1]$ measures the strength of the time-reversal symmetry breaking. The relation between $\alpha$ and the magnetic flux $\Phi$ through a two-dimensional billiard (area $A$, no impurities, Fermi velocity $v_{\mathrm{F}}$ ) is [8,9]

$$
M \alpha^{2}=c(\Phi e / h)^{2} \hbar v_{\mathrm{F}}\left(A \delta^{2}\right)^{-1 / 2},
$$

with $c$ a numerical coefficient of order unity. (For example, $c=\frac{2}{3} \sqrt{\pi}$ for a circular billiard which is chaotic because of diffuse boundary scattering [9.) Time-reversal symmetry is effectively broken when $M \alpha^{2} \simeq N$, which occurs for $\Phi \ll h / e$. The effect of such weak magnetic fields on the bulk superconductor can be ignored.

A key step [10] is to write the determinantal equation (2) as an eigenvalue equation for an effective Hamiltonian $H_{\text {eff }}$,

$$
\begin{aligned}
& \operatorname{Det}\left(E-H_{\mathrm{eff}}\right)=0, \\
& H_{\mathrm{eff}}=\left(\begin{array}{cc}
H & -\pi W e^{i \phi} W^{\mathrm{T}} \\
-\pi W e^{-i \phi} W^{\mathrm{T}} & -H^{*}
\end{array}\right) .
\end{aligned}
$$

The average density of states $\rho(E)$ of the chaotic billiard results from

$\rho(E)=-\pi^{-1} \operatorname{Im} \operatorname{Tr} \mathcal{G}\left(E+i 0^{+}\right), \quad \mathcal{G}(z)=\left\langle\left(z-H_{\mathrm{eff}}\right)^{-1}\right\rangle$,

where $\langle\cdots\rangle$ denotes an average of $H$ with distribution

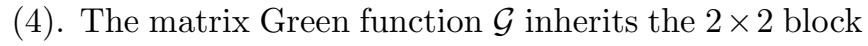
structure from the effective Hamiltonian $H_{\text {eff }}$. Because of this block structure, it is convenient to define the $2 \times 2$ Green function

$$
G=\left(\begin{array}{ll}
G_{11} & G_{12} \\
G_{21} & G_{22}
\end{array}\right)=\frac{\lambda}{M}\left(\begin{array}{ll}
\operatorname{Tr} \mathcal{G}_{11} & \operatorname{Tr} \mathcal{G}_{12} \\
\operatorname{Tr} \mathcal{G}_{21} & \operatorname{Tr} \mathcal{G}_{22}
\end{array}\right)
$$

Using the diagrammatic method of Refs. 11] and [12], adapted for a matrix Green function, we have derived a self-consistency equation for $G$. To highest order in $1 / M$, this equation reads

$$
G=\frac{\lambda}{M} \sum_{n=1}^{M}\left(\begin{array}{cc}
z-\lambda G_{11} & \pi w_{n}^{2}+x \lambda G_{12} \\
\pi w_{n}^{* 2}+x \lambda G_{21} & z-\lambda G_{22}
\end{array}\right)^{-1},
$$

where we have abbreviated $w_{n}^{2}=\left(W e^{i \phi} W^{\mathrm{T}}\right)_{n n}$ and $x=$ $\left(1-\alpha^{2}\right) /\left(1+\alpha^{2}\right)$. Eq. (9) is complemented with the boundary condition $G \rightarrow \lambda / z$ for $|z| \rightarrow \infty$.

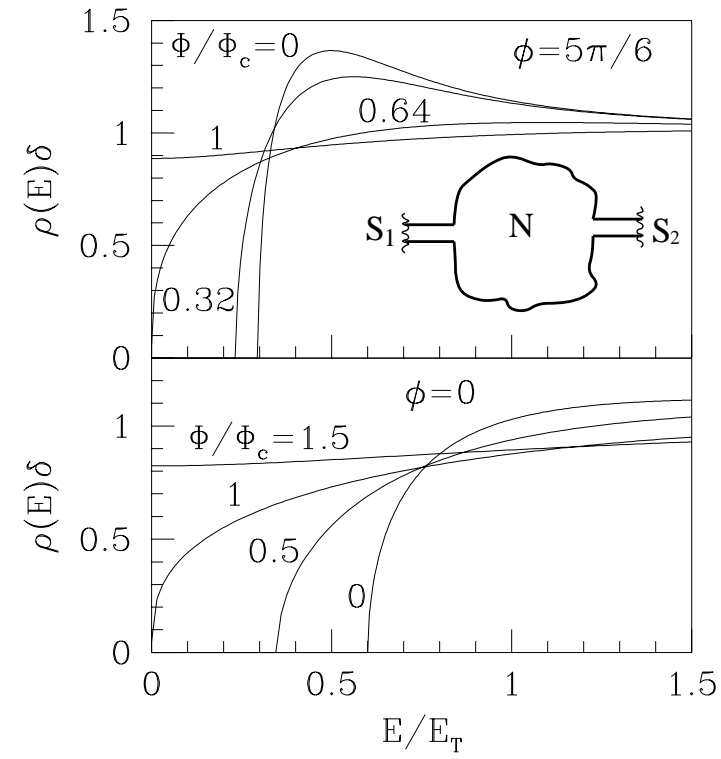

FIG. 1. Density of states of a chaotic billiard coupled to two superconductors by identical ballistic point contacts, for four values of the magnetic flux $\Phi$ through the billiard. The phase difference $\phi$ between the superconductors equals $5 \pi / 6$ and 0 in the top and bottom panel, respectively. The curves are computed from Eqs. (月) and (10). The Thouless energy is given by $E_{\mathrm{T}}=N \delta / 2 \pi$, and the critical flux $\Phi_{\mathrm{c}}$ is defined by Eqs. (同) and (11).

We take the limit $M \rightarrow \infty$ at fixed $M \alpha^{2}$ and $\delta$, and assume in addition that $N \gg 1$. Eq. (9) then simplifies to

$$
\begin{aligned}
G_{11}= & {\left[\frac{1}{2}\left(\Phi / \Phi_{\mathrm{c}}\right)^{2} G_{11}-\pi z / N \delta\right] \times } \\
& {\left[G_{12}^{2}+G_{12} / \cos (\phi / 2)\right], } \\
G_{22}= & G_{11}, G_{21}=G_{12}, G_{12}^{2}=1+G_{11}^{2},
\end{aligned}
$$

where we have defined the critical flux $\Phi_{c}$ by

$$
M \alpha^{2}=\frac{1}{8} N\left(\Phi / \Phi_{\mathrm{c}}\right)^{2} .
$$

The solution of Eq. (10) for $\phi=0$ and $\phi=5 \pi / 6$ is plotted in Fig. 目 for several values of $\Phi / \Phi_{\mathrm{c}}$. For $\Phi=0$ and $\phi=0$ the excitation gap equals $E_{\text {gap }}=a E_{\mathrm{T}}$, with $E_{\mathrm{T}}=N \delta / 2 \pi$ and $a=2^{-3 / 2}(\sqrt{5}-1)^{5 / 2} \approx 0.6$. The gap decreases with increasing flux $\Phi$ or phase difference $\phi$. When $\phi=0$, the gap closes at the critical flux $\Phi_{\mathrm{c}} \simeq$ $(h / e)\left(N \delta / \hbar v_{\mathrm{F}}\right)^{1 / 2} A^{1 / 4}$. When $\phi=\pi$, there is no gap at any magnetic field. For $\phi$ between 0 and $\pi$, the gap closes at the flux $\Phi_{\mathrm{c}}(\phi)$ given by

$$
\Phi_{\mathrm{c}}(\phi)=\left[\frac{2 \cos (\phi / 2)}{1+\cos (\phi / 2)}\right]^{1 / 2} \Phi_{\mathrm{c}} .
$$

So far we have used random-matrix theory to describe the chaotic system. As a test, we can compute the exact quantum mechanical density of states of a specific 


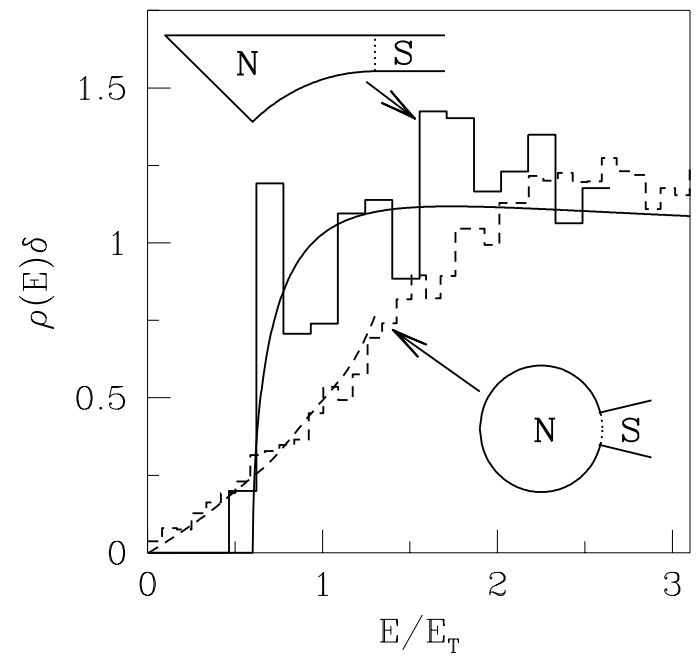

FIG. 2. Histograms: density of states of a billiard coupled to a superconductor, computed from Eq. (1) and averaged over a range of Fermi energies. A chaotic Sinai billiard (top inset, solid histogram) is contrasted with an integrable circular billiard (bottom inset, dashed histogram). The number of propagating transverse modes at the normal-metal-superconductor interface (dotted line in the insets) equals $N=20$ in the chaotic billiard and $N=30$ in the circular billiard. The solid curve is the prediction from random-matrix theory, the dashed curve is the prediction from the Bohr-Sommerfeld approximation.

billiard, coupled to a superconductor. Following Doron, Smilansky, and Frenkel 13 we study a segment of a Sinai billiard, drawn to scale in the top inset of Fig. 2. The scattering matrix $S_{0}(E)$ is determined by matching wave functions at the dotted line separating the billiard (area $A$ ) from the lead (width $W$ ). The NS interface is also chosen at the dotted line. The density of states $\rho(E)$ follows from Eq. (11). We average $\rho(E)$ over a small variation of $E_{\mathrm{F}}$, such that the number of modes $N=\operatorname{Int}\left[m v_{\mathrm{F}} W / \pi \hbar\right]$ in the lead does not change. The result for $N=20$ is shown in Fig. 2 (solid histogram), and is seen to agree quite well with the prediction from random-matrix theory (solid curve). There is no adjustable parameter in this comparison, the mean level spacing $\delta$ following directly from $\delta=\pi \hbar^{2} / m A$.

We now turn from a chaotic to an integrable billiard. In Ref. [4], we computed the density of states of a rectangular billiard, coupled to a superconductor by a narrow lead, and found a linearly vanishing $\rho(E)$ for small $E$. Here, we present data for a circular billiard (radius $R$ ) in support of our claim that the absence of an excitation gap is generic for integrable billiards. The circular billiard considered is drawn to scale in the bottom inset of Fig. 2. The scattering matrix $S_{0}(E)$ is again deter- mined by matching wave functions at the dotted line, which also determines the location of the NS interface. A wedge-shaped lead (opening angle $\theta$ ) is chosen in order not to break the rotational symmetry (which simplifies the calculations). The density of states is averaged over a range of Fermi energies at fixed $N=\operatorname{Int}\left[m v_{\mathrm{F}} R \theta / \pi \hbar\right]$. The result for $N=30$ is the dashed histogram in Fig. 2 .

The density of states in the integrable billiard can be approximated with the Bohr-Sommerfeld quantization rule 沟,

$$
\rho_{\mathrm{BS}}(E)=N \int_{0}^{\infty} d s P(s) \sum_{n=0}^{\infty} \delta\left(E-\left(n+\frac{1}{2}\right) \pi \hbar v_{\mathrm{F}} / s\right) .
$$

Here $P(s)$ is the classical probability that an electron entering the billiard will exit after a path length $s$. We have calculated $P(s)$ for the circular billiard shown in Fig. 2, by generating a large number of classical trajectories. The resulting density of states $\rho_{\mathrm{BS}}(E)$ is in good agreement with the quantum mechanical result in Fig. 2 .

To conclude, we have calculated the density of states of a chaotic Sinai billiard, connected to a superconductor. The result is in good agreement with the prediction from random-matrix theory [4]. The excitation gap closes at a critical flux $\Phi_{\mathrm{c}}$ through the billiard. In order of magnitude, $\Phi_{\mathrm{c}} \simeq(h / e)\left(\tau_{\text {ergodic }} / \tau_{\text {dwell }}\right)^{1 / 2}$, where $\tau_{\text {dwell }}$ is the mean dwell time of an electron in the billiard and $\tau_{\text {ergodic }}$ is the time required to explore the entire available phase space. The precise value of $\Phi_{c}$ depends on the shape of the billiard, but the dependence on the tunnel probability $\Gamma$ and the phase difference $\phi$ is universal:

$$
\Phi_{\mathrm{c}}(\Gamma, \phi)=\Phi_{\mathrm{c}}(1,0)\left[\frac{2 \cos (\phi / 2)}{\cos (\phi / 2)-1+2 / \Gamma}\right]^{1 / 2} .
$$

We have shown that the excitation gap, generic for chaotic billiards, is absent in an integrable circular billiard. Instead, the density of states $\rho(E) \propto E$ for small $E$, as in the rectangular billiard considered previously [ $[$. The agreement with the semi-classical Bohr-Sommerfeld approximation is better for the circular than for the rectangular billiard. A rigorous semi-classical theory for this problem remains to be developed.

This work was supported by the Dutch Science Foundation NWO/FOM and by the Human Capital and Mobility program of the European Community.

[1] O. Bohigas, M.-J. Giannoni, and C. Schmit, Phys. Rev. Lett. 52, 1 (1984).

[2] M. V. Berry and M. Tabor, Proc. R. Soc. London A 356, 375 (1977); M. V. Berry, Proc. R. Soc. London A 400, 229 (1985). 
[3] M. L. Mehta, Random Matrices (Academic, New York, 1991).

[4] J. A. Melsen, P. W. Brouwer, K. M. Frahm, and C. W. J. Beenakker, Europhys. Lett. 35, 7 (1996).

[5] C. W. J. Beenakker, Phys. Rev. Lett. 67, 3836 (1991); 68, 1442(E) (1992). For a review, see C. W. J. Beenakker, in Transport Phenomena in Mesoscopic Systems, edited by H. Fukuyama and T. Ando (Springer, Berlin, 1992).

[6] C. Mahaux and H. A. Weidenmüller, Shell-Model Approach to Nuclear Reactions (North-Holland, Amsterdam, 1969).

[7] A. Pandey and M. L. Mehta, Commun. Math. Phys. 87, 449 (1983).

[8] O. Bohigas, M.-J. Giannoni, A. M. Ozorio de Almeida, and C. Schmit, Nonlinearity 8, 203 (1995).

[9] K. M. Frahm and J.-L. Pichard, J. Phys. I France 5, 847 (1995).

[10] K. M. Frahm, P. W. Brouwer, J. A. Melsen, and C. W. J. Beenakker, Phys. Rev. Lett. 76, 2981 (1996).

[11] A. Pandey, Ann. Phys. (N.Y.) 134, 110 (1981).

[12] E. Brézin and A. Zee, Phys. Rev. E 49, 2588 (1994).

[13] E. Doron, U. Smilansky, and A. Frenkel, Physica D 50, 367 (1991); E. Doron and U. Smilansky, Phys. Rev. Lett. 68, 1255 (1992). 